\title{
Motor control deficits of orofacial muscles in cerebral palsy
}

\author{
CHRISTOPHER W VAUGHAN, PETER D NEILSON, NICHOLAS J O'DWYER \\ From the Spastic Centre Research Unit, Department of Neurology, The Prince Henry Hospital and School of \\ Medicine, University of New South Wales, Australia
}

SUMMARY Voluntary control of the masseter and orbicularis oris superioris muscles was examined in able bodied and cerebral palsied subjects using visual tracking tasks. A smoothed measure of muscle activity (the full-wave rectified and low-pass filtered electromyogram) was presented as a marker on a computer display screen and the subjects could control the vertical position of the marker by voluntarily altering the level of isometric contraction of one of the muscles. A target marker was also displayed on the screen and the subjects were required to follow or "track" the irregular movements of this target with the response marker. Their success in aligning the response marker with the target was analysed for these orofacial muscles. The masseter is influenced by muscle spindle based reflexes, while the orbicularis oris superioris lacks such reflex control. The cerebral palsied subjects displayed similarly poor control over both muscles, implying that their voluntary motor deficits are not related to abnormal muscle spindle based reflexes. It is suggested that the impairment may be related to perceptual-motor integration.

The prevailing theories concerning motor dysfunctions in cerebral palsy generally are dependent upon the disruptive effect of abnormal reflexes on motor control. ${ }^{1-4}$ While the pathophysiology of cerebral palsy involves voluntary deficits as well as the loss of reflex control ${ }^{25}$ it is the latter which is used to categorise the motor dysfunction. Recently, it has been suggested that the major deficit may not be abnormal reflex activity imposing restraints on posture and movement, but that all descending motor commands, including reflex control and muscle contraction signals, are inappropriate. ${ }^{5-9}$ In the absence of reflex effects, motor control is thought to be exceedingly poor and not due solely to damage to the descending pyramidal tract. ${ }^{7-9}$

Evidence supporting the proposition that descending motor commands in cerebral palsy are inappropriate has been based on observing motor control after removal of reflex constraints. For example, the suppression of spinal reflexes by anti-spastic drugs such as baclofen and phenol does not parallel any improvement in motor performance. ${ }^{71011}$ Simi-

Address for reprint requests: Peter D Neilson, Spastic Centre Research Unit, The Prince Henry Hospital, Little Bay, N.S.W., 2036 Australia.

Received 6 October 1987.

Accepted 1 December 1987 larly, cerebral palsied subjects have been trained to control and reduce tonic stretch reflex activity but show no associated improvement in motor control? suggesting that spasm and spasticity are not the primary cause of the dysfunction. ${ }^{8}$

Other evidence may be obtained by observing orofacial muscles with and without reflex activity. The masseter, a jaw closing muscle, is richly innervated by muscle spindles ${ }^{12}$ and exhibits stretch reflex activity. ${ }^{13}$ Lip muscles such as the orbicularis oris superioris have few, if any, spindle endings. ${ }^{14}$ This muscle displays phasic reflex activity in response to tapping and similar intermittent stimuli. ${ }^{15}{ }^{16}$. However, during normal speech and non-speech motor tasks, which are limited to frequencies below $15 \mathrm{~Hz},{ }^{17}$ no tonic stretch reflex activity is observed. ${ }^{18}$ Voluntary control of these two muscles, therefore, may be expected to be differentially affected by tonic stretch reflex abnormalities. All other factors being equal, cerebral palsied subjects should exhibit very poor control over the masseter due to reflex abnormalities, while control of orbicularis oris superioris should be free of such abnormalities.

Tracking tasks based on dynamic control simulate everyday motor behaviour and have been used extensively in measuring both motor skill and human control characteristics in man-machine systems. ${ }^{19-21}$ In the present study, a visual pursuit tracking task was devised in which performance was dependent on vol- 
untary control of the isometric contraction level of either masseter or orbicularis oris superioris. Thus, voluntary control of these muscles could be compared.

\section{Methods}

In the tracking task employed here, a target marker and a response marker are presented simultaneously on a display screen. The target moves irregularly in the vertical plane and the subject is required to attempt to keep the two markers aligned by following or "tracking" the target with the response marker. For the present experiment, the vertical position of the response marker on the screen is controlled by the isometric contraction of a single muscle, either masseter or orbicularis oris superioris.

The contraction level of the muscle was measured via the electromyogram (EMG) from the muscle. The EMG, recorded with surface electrodes located over the muscle, was amplified, full-wave rectified and low-pass filtered $(1 \mathrm{~Hz}$ cut-off frequency). This smoothed EMG (IEMG) signal was used to control the position of the response marker on the display screen, whereby an increase or decrease in IEMG drove the response marker up or down the display screen, respectively. It should be noted that although activity was monitored only from a single muscle, tracking performance did not require selective activation of that muscle. Indeed, the subjects were free to co-contract synergistic, antagonistic or any more distant muscles. For each subject, the gain of the EMG amplifier was adjusted so that the range of deflection of the response marker on the display screen corresponded to the range between $30-60 \%$ of maximal voluntary isometric contraction of the muscle. Thus, the tracking task assessed control over the mid-range of contraction. Subjects generated activity in masseter by clenching the jaw and then increasing and decreasing the bite force. Activity in orbicularis oris superioris was generated by pressing the lips together or by compressing the upper lip against the upper incisors and then varying the lip pressure in either case.

A random signal was computer generated and smoothed by a second order low-pass Butterworth digital filter which had a cut-off frequency of $0.35 \mathrm{~Hz}$. This filtered signal was used to drive the target on the display screen. Both the target and the response marker positions were sampled and displayed at $5 \mathrm{~Hz}$. These sampled signals were also stored as the "stimulus" and "response" signals for subsequent analysis. The EMG filtering removed all signal power above half this sampling rate, so that distortion of the signal due to aliasing was avoided. 2223

Responses from orbicularis oris superioris and masseter muscles were recorded for 100 seconds in 10 cerebral palsied subjects (aged between 19 and 32 years) and 10 able bodied subjects (aged between 20 and 34 years). To eliminate transfer effects, the order of muscle recording was randomised in each subject group. The cerebral palsied subjects exhibited clinically mixed signs of both spasticity and athetosis, with a greater predominance of one or the other in individual cases. All these subjects displayed increased jaw jerks and their dysarthric speech was judged at best poorly intelligible. These subjects did not display any other symptoms such as epilepsy, or sensory deficits, which are commonly associated with the condition. All subjects gave informed consent to the experimental procedure and could understand the task to be performed.

\section{Analysis technique}

The stored stimulus and response signals were analysed via the quasi-linear technique, which provides a comprehensive description of human tracking behaviour. ${ }^{19-21}$. It is based on linear spectral analysis using the correlation method. ${ }^{2223}$ This analysis effectively separates the subject's response signal into a coherent component which is linearly correlated with the movement of the target and an incoherent component (known as the "remnant") which is not correlated with the target. The ratio of the linear component to the total variance of the subject's response is quantified by the "coherence square function". This measures the proportion of the response variance at any frequency which is linearly related to the target at that frequency. The remaining proportion of the response at that frequency is remnant. The coherence square function is analogous to the square of the correlation coefficient (the coefficient of determination, $r^{2}$ ) in a regression analysis.

It can be shown mathematically and experimentally ${ }^{24}$ that all response marker movements which are not coherent with (that is, linearly related to) the target always add to the error signal. Even if the subject employs a non-linear tracking strategy, it is only that part of the response described by the best fit linear relation which can reduce the tracking error. Thus, the remnant always adds to the error. Sources of remnant in the present study could include such factors as nonlinear (including time-varying) tracking strategies, muscle spasm, EMG noise and so on.

The best fit linear relation between the target and the coherent response is described by gain and phase frequency response curves. The gain describes the amplitude and the phase describes the timing of the subject's tracking response relative to the target. Gain, phase, and remnant frequency response curves thus provide objective measures of tracking behaviour, whether linear or non-linear, and as such provide a detailed assessment of the quality of tracking performance. For perfect tracking, the gain must approach unity, the phase lag must approach zero, and there must be no remnant. These measures reveal tracking strategies employed by the subject and enable the source of the subject's errors (for example, prolonged reaction time, overshoot, involuntary spasm, etc.) to be elucidated. Although the analysis technique is described as "quasi-linear", it is a most appropriate tool for obtaining a quantitative assessment of tracking behaviour whether linear or non-linear. ${ }^{19} 21$

Mean values of the coherence square function, total response power (or variance), and remnant response power were computed for both muscles in each subject group. The gain and phase lag were calculated only for the able bodied subjects. In the cerebral palsied subjects the linear response was small compared to the remnant response, resulting in low coherence values. The gain and phase curves cannot be estimated reliably when the coherence is low.

The power spectrum of the stimulus signal decreased to negligible values by $0.6 \mathrm{~Hz}$ (fig 1). Responses at, or above, this frequency can not be linearly related to the stimulus because there is too little stimulus signal power for a significant correlation. Therefore, a maximum frequency of 


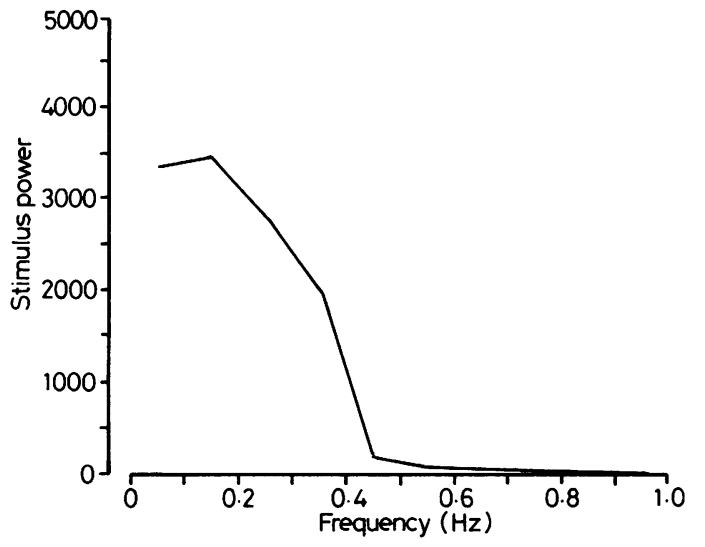

Fig 1 Power spectrum of stimulus signal. Power in all spectra is in arbitrary units. Frequency in $\mathrm{Hz}$.

$0.55 \mathrm{~Hz}$ was considered for the coherence square function, gain, and phase lag. Spectra of the total response power and of the remnant power, however, were considered for frequencies up to $0.95 \mathrm{~Hz}$, since the frequency bandwidth of these signals, especially the remnant, was not necessarily limited to $0.55 \mathrm{~Hz}$. Above $0.95 \mathrm{~Hz}$, response power dropped to insignificant levels as a result of EMG filtering. A spectral spacing of $0 \cdot 1 \mathrm{~Hz}$ was chosen as a compromise to reduce spectral errors while maintaining some frequency resolution given the sample length and sampling rate constraints.

A three factor analysis of variance (ANOVA), with repeated measures, compared subject group performance (factor 1) for both muscles (factor 2) over the frequency band (factor 3 ) tested. ${ }^{25}$ In addition, a two-factor ANOVA was also carried out separately on each subject group. The total response power, remnant power, and coherence were first logarithmically transformed. This resulted in normally distributed spectral values and satisfied the condition of homogeneity of within-cell variance required for the ANOVA procedure. ${ }^{25}$

\section{Results}

\section{Subject group comparisons}

Direct observation of the tracking runs in fig 2 indicates that motor control in the cerebral palsied subjects was inferior to the able bodied subjects. The able bodied subjects followed the target with a time delay but the waveform of their response was similar to that of the stimulus. The cerebral palsied subjects maintained the correct range of response and varied their responses with sufficient speed. However, their responses were poorly related to the stimulus, so that they generally failed to reproduce the stimulus waveform.

The relative root mean square (rms) error provides an overall index of tracking ability. ${ }^{19}$ It is obtained by expressing the ratio of the rms error and rms stimulus values as a percentage. Application of Student's $t$ test $^{25}$ confirmed the significantly poor control over both muscles in the cerebral palsied subjects compared with the able bodied subjects $(p=0.001)$. However, there was no significant difference between masseter (relative rms error, 247\%) and orbicularis oris superioris (relative rms error, $260 \%$ ) for the cerebral palsied group ( $p>0 \cdot 1$.) Similarly, no difference could be detected between masseter (relative rms error, $118 \%$ ) and orbicularis oris superioris (relative rms error $114 \%$ ) for the able bodied subjects $(\mathrm{p}>0 \cdot 1)$.

The poor tracking in the cerebral palsied subjects could not be ascribed to an inability to understand the nature of the task. They could appreciate precisely what was required of them and could explain what they were attempting to do. It was evident (see fig 2) that they could activate and relax both muscles and could modulate the contraction level over the appropriate range for the tracking task. In fact, as can be seen in fig $3 \mathrm{a}$, the cerebral palsied subjects produced a dynamic response which was much larger in power than that of the stimulus. Moreover, this total response power was much larger than that of the able bodied subjects (subject factor, $p<0.001$ ), whose response power was similar to the stimulus power.

Coherence for the cerebral palsied subjects was much lower than that for the able bodied subjects (subject factor, $\mathrm{p}<0.001$; fig 3b). Coherence for the cerebral palsied subjects remained below 0.2 for all frequencies, indicating that only a small proportion $(<20 \%)$ of their response was linearly related to the stimulus. The largely remnant responses of the cerebral palsied subjects (fig 3c) add completely to the error. In contrast, the able bodied subjects had coherence values of about 0.6 up to $0.25 \mathrm{~Hz}$, above which they dropped steadily with increasing frequency (fig 3b). A much larger proportion of their response power, therefore, was linearly related to the simulus. Similarly, their remnant response power was significantly less than that for the cerebral palsied subjects (subject factor, $p<0.001$; fig $3 c$ ).

\section{Muscle group and frequency band comparisons}

All power spectra varied significantly with frequency (frequency factor, $p<=0.01$ ) and these frequency characteristics varied differently over the two subject groups (subject-frequency interaction, $\mathrm{p}<0.001$ ). No significant difference was detected between masseter and orbicularis oris superioris in total response power, coherence, or remnant power (muscle factor, $p>0.55)$. Moreover, the frequency characteristics did not differ between the two muscles (muscle-frequency interaction, $\mathrm{p}>0.22$ ).

\section{Cerebral palsied subjects}

In the two factor ANOVA on the cerebral palsied 
(a) Able bodied subject

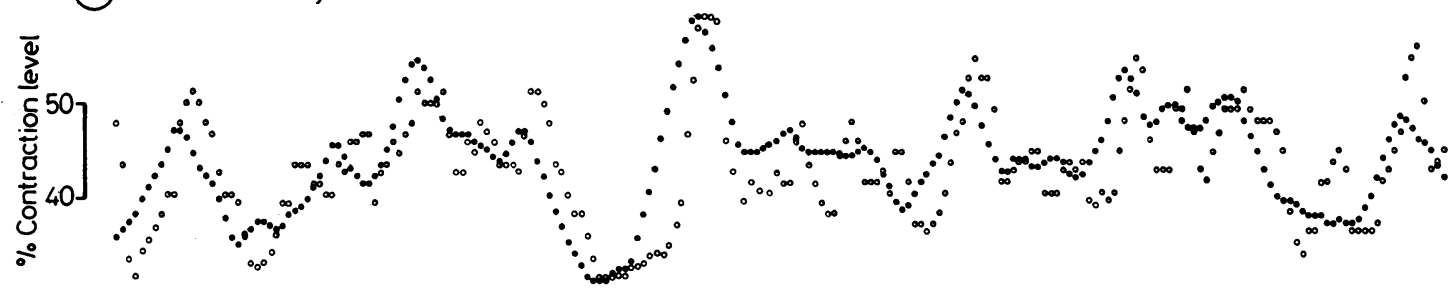

(b) Cerebral palsied subject
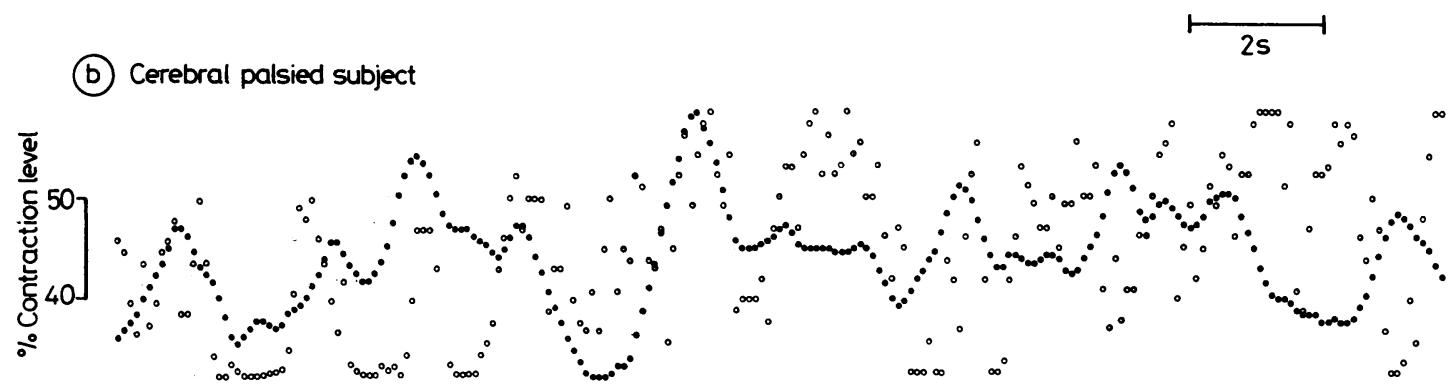

Fig 2 Typical plots of the subjects' responses (empty circles) to the stimulus (filled circles) during tracking for (a) able bodied, and $(b)$ cerebral palsied subjects. Vertical calibration represents $40 \%$ and $50 \%$ of maximum contraction. The traces show a 20-second segment of tracking with masseter.

subjects the coherence was found to be lower for orbicularis oris superioris than masseter, but the difference was not statistically significant (muscle factor, $p=0.08$; fig $3 b$ ). The total response and remnant were marginally greater for the orbicularis oris superioris than masseter, as seen in figs $3 a$ and $c$, but again these differences were not significant (muscle factor, $p=0.09,0.08$ respectively). The total response and remnant varied with frequency (frequency factor, $\mathrm{p}<0.001$ ), but the coherence was fairly constant across frequencies (frequency factor, $p=0.53$; fig $3 b$ ). The two muscles had similar frequency characteristics for all spectra, so that the muscle-frequency interactions were not significant $(p>0 \cdot 35)$.

\section{Able bodied subjects}

The two factor analysis of the able bodied subjects revealed a small difference in total response and a larger difference in coherence between the two muscles (muscle factor, $p=0.05,0.03$ respectively; fig $3 a, b$ ), while the remnant was similar for both muscles (muscle factor, $p=0.45$; fig $3 \mathrm{c}$ ). The frequency characteristics for the total response were similar for both muscles (muscle-frequency interaction, $p=0.49$ ), but varied between the two muscles for the coherence and remnant (muscle-frequency interaction, $p=0.04$, 0.03 respectively).

As can be seen in fig $4 \mathrm{a}$, the gain was similar for both muscles (muscle factor, $p=0.4$ ) and it varied similarly with frequency in both muscles (musclefrequency interaction, $p=0 \cdot 8$ ). The phase lags, on the other hand, were different in the two muscles, as can be seen in fig $4 b$ (muscle factor, $p<0.001$ ). The variation of phase with frequency, however, did not differ significantly between the two muscles (musclefrequency interaction, $p=0 \cdot 25$ ).

\section{Discussion}

The total response power of the cerebral palsied subjects covered a similar frequency range to that of the able bodied subjects but was of much greater magnitude. It is evident, therefore, that the problem in the cerebral palsied subjects is not an inability to activate the muscles nor to vary the level of activity. The timing and gradation of these variations in level of activity, however, are inappropriate to achieve the subject's goal, in this case, to keep the response marker aligned with the target. Muscle spasms which resulted in contractions outside the prescribed range of $30-60 \%$ of maximum voluntary contraction (causing the response marker to be maintained at the extreme of its range of deflection) were present in eight of the 20 cerebral palsied tracking runs and clearly disrupted tracking control. Such spasms, however, accounted for less than $4 \%$ of the total error and tracking control was still very poor in their absence. The primary abnormality appears to consist of inappropriate voluntary activity rather than involuntary contractions. This lack of voluntary control is reflected in the fact that their muscle response contained variations of the same frequency and amplitude as the stimulus but nevertheless, were poorly related to the stimulus. 


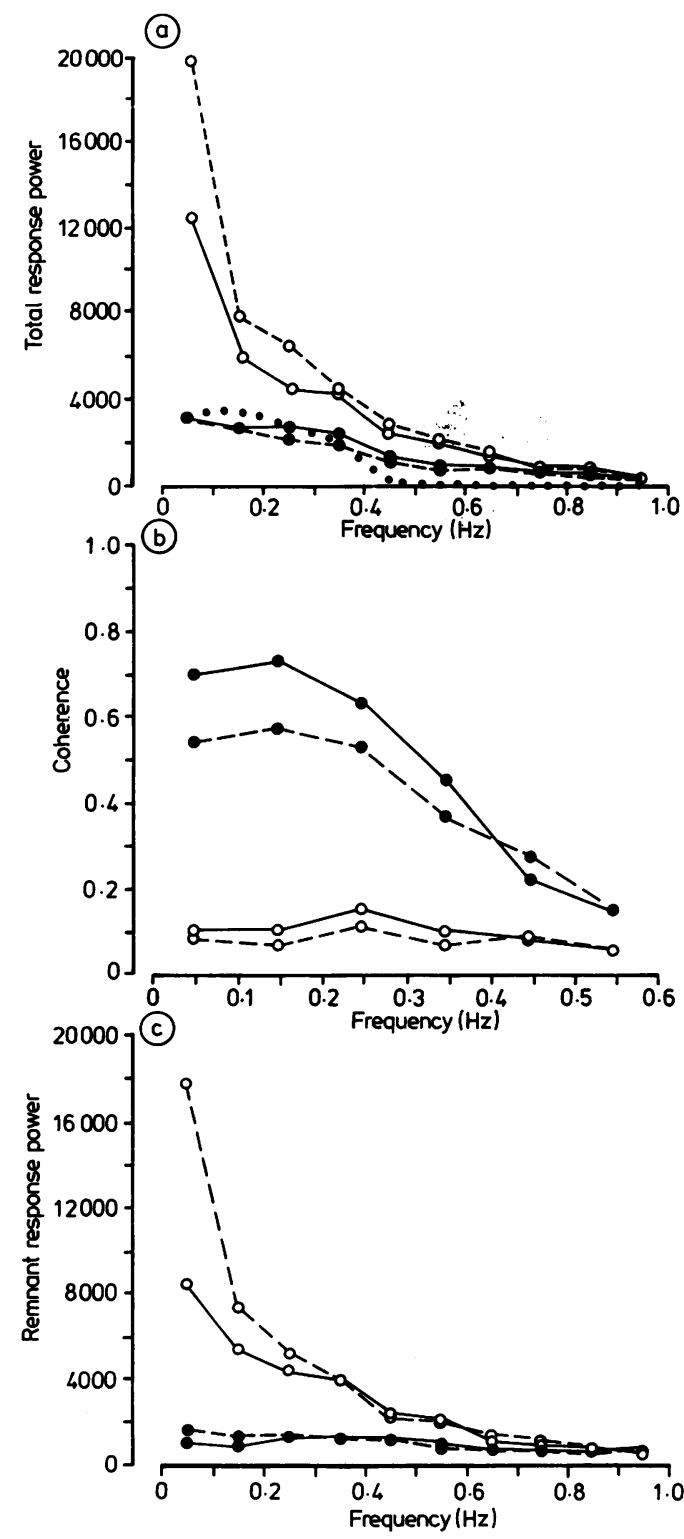

Fig 3 (a) Total response power, (b) Coherence square function, and (c) Remnant response power, against frequency, for masseter (solid line) and orbicularis oris superioris (dashed line), in the cerebral palsied (o) and able bodied (O) subjects. Stimulus signal power is also shown in (a) (dotted line). Frequency in $\mathrm{Hz}$; power in arbitrary units, coherence has no units. All curves are averaged across the 10 subjects in each group.

Since tonic stretch reflexes were not involved in the control of orbicularis oris superioris during the tracking task, the motor dysfunction in this muscle in the cerebral palsied subjects cannot be attributed to
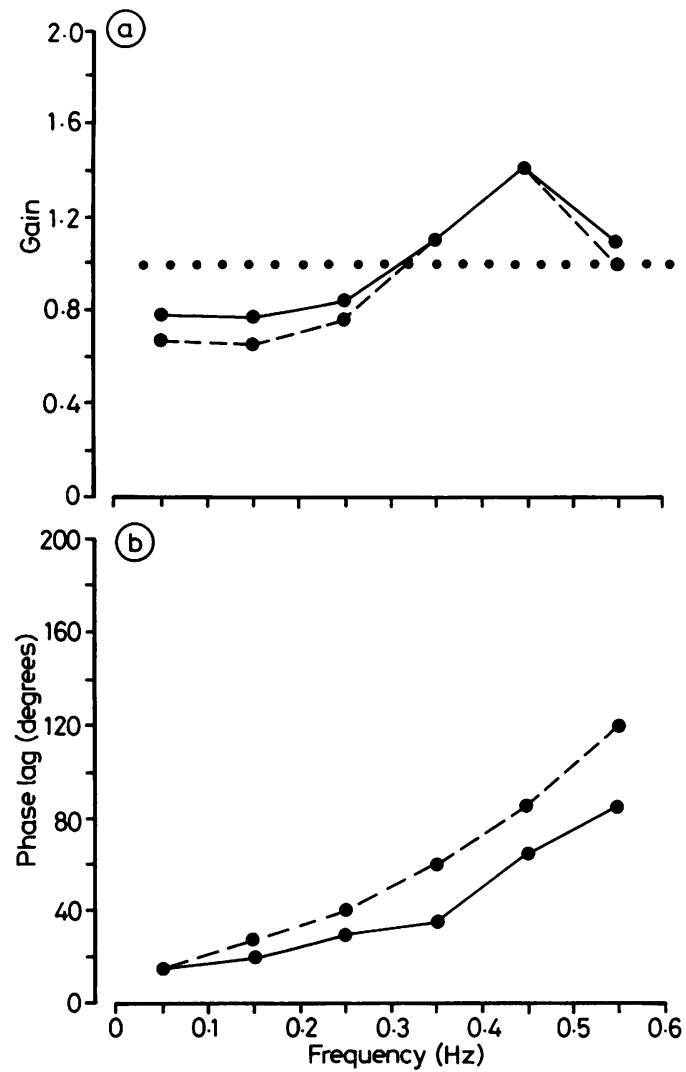

Fig 4 (a) Gain, and (b) Phase lag, against frequency, for masseter (solid line) and orbicularis oris superioris (dashed line) in the able bodied subjects. Frequency in $\mathrm{Hz}$, Gain has no units, Phase lag in degrees. All curves are averaged across the 10 able bodied subjects.

abnormal reflexes. The same is probably true of masseter, despite the presence of tonic stretch reflexes in this muscle, since the controlled variable in the present study was IEMG during isometric contractions. The use of isometric IEMG activity minimises stretch reflex input. Moreover, the influence of adjacent muscles and the effects of orofacial biomechanics also are minimised in this experimental paradigm. Thus, isometric IEMG activity provides a relatively direct measure of voluntary control of a single muscle. In any event, whatever the role of tonic stretch reflexes in masseter, its control was no poorer than that of orbicularis oris superioris. If anything, the trend was in the opposite direction in the cerebral palsied subjects. Similar findings were reported by Barlow and Abbs, ${ }^{26}$ who compared motor impairment in lip, tongue, and jaw muscles in cerebral palsied subjects using force stability during steady isometric contractions as the controlled variable. 
Although their muscle responses covered a similar frequency range, the two groups nevertheless displayed different frequency characteristics, or dynamic properties. That is, the distribution of total response power, remnant power, and coherence across frequencies differed between the groups (fig 3). This suggests that the dysfunction in the cerebral palsied subjects was altering physiological processes underlying voluntary control of isometric tension. Since both muscles displayed similar frequency characteristics within each group, the same mechanism was likely to be producing the dysfunction in both muscles in the cerebral palsied subjects, despite the presence of tonic stretch reflexes in one of the muscles and their absence in the other.

In the able bodied subjects, control of masseter was slightly superior to that of orbicularis oris superioris. This superiority was most evident in the smaller phase lag for masseter. The phase lag reflects the time required to analyse sensory information and to formulate an appropriate motor response. Practice or training leads to a reduction in this phase lag, as well as an increase in the gain towards unity and a smaller remnant. ${ }^{1927}$ Able bodied subjects have extensive experience in controlling jaw closing tension during biting and chewing, whereas lip pressure control as employed here is a more novel task. This may account for the superior performance of masseter.

The similarly poor control of orbicularis oris superioris and masseter in the cerebral palsied subjects clearly fails to support a predominant role for stretch reflex abnormalities in determining their motor impairment and this illustrates the dissociation which can occur between the level of spasticity and voluntary control deficit in these subjects. This result extends the findings or earlier studies which reported similar conclusions. ${ }^{5-9} 1826$ In light of these findings, the physiological processes underlying voluntary control of movement perhaps may assume a predominant role. We have proposed previously ${ }^{9}$ that the primary abnormality underlying the motor impairment in cerebral palsy is an inability to learn the relationships between the motor commands to muscles and the resulting perceptual consequences of the movement. Such perceptual-motor learning forms the basis of movement control and the aquisition of motor skill. Its disruption results, not in an inability to move, but in a lack of control of movement. The poorly controlled muscle responses observed in the present study are consistent with this proposal.

This study was supported by the National Health and Medical Research Council of Australia. We are grateful to Mr Neil McLeod, Mr Barrie Dentice, and the members of the board of the Spastic Centre of N.S.W. for their support. We thank Mr J Perini for generous financial assistance.

\section{References}

1 Narabayashi H, Nagahata $M$, Nagao T, Shimazu H. A new classification of cerebral palsy based upon neurophysiologic considerations. Confin Neurol 1965;25:378-92.

2 Brain WR. Brain's Diseases of the Nervous System. Revised by Walton JN, 8th ed. Oxford: Oxford University Press, 1977.

3 Low NL. Cerebral palsy. In: Rudolph AM, Barnett HL, Einhorn AH, eds. Pediatrics, 16th ed. New York: Appleton-CenturyCrofts, 1977:1779-83.

4 Mountcastle VB. Medical Physiology. 14th ed. 1980, St Louis: Mosby.

5 Levitt S. Treatment of Cerebral Palsy and Motor Delay. 2nd ed., Philadelphia: Blackwell Scientific Publ., 1982.

6 Sahrmann SA, Norton BJ. Relationship of voluntary movement to spasticity in the upper motor neuron syndrome. Ann Neurol 1977;2:460-5.

7 McLellan DL. Co-contraction and stretch reflexes in spasticity during treatment with Baclofen. J Neurol Neurosurg Psychiatry 1977;40:30-8.

8 Neilson PD, McCaughey J. Self-regulation of spasm and spasticity in cerebral palsy. J Neurol Neurosurg Psychiatry 1982; 45:320-30.

9 Neilson PD, O'Dwyer NJ. Pathophysiology of dysarthria in cerebral palsy. J Neurol Neurosurg Psychiatry 1981;44:1013-9.

10 Nathan PW. Treatment of spasticity with perineural injections of phenol. Dev Med Child Neurol 1969;11:384.

11 Landau WM. Spasticity: the fable of a neurological demon and the emperor's new therapy. Arch Neurol 1974;31:217-9.

12 Cooper S. Muscle spindles and other muscle receptors. In: Bowen $\mathrm{GH}$, ed. The Structure and Function of Muscles. New York: Academic Press 1960:381-420.

13 Lamarre Y, Lund JP. Load compensation in human Masseter muscles. J Physiol (Lond) 1975;253:21-35.

14 Lovell M, Sutton D, Linderman RC. Muscle spindles in nonhuman primate's extrinsic auricular muscles. Anat Res 1977;189:519-24.

15 Larson CR, Folkins LW, McClean MD, Muller EM. Sensitivity of the human perioral reflex to parameters of mechanical stretch. Brain Res 1978;146:159-64.

16 Folkins JW, Larson CR. In search of a tonic vibration reflex in the human lip. Brain Res 1978;151:409-12.

17 Muller E, Abbs J, Kennedy J. Some systems physiology considerations for vocal control. In: Stevens, $\mathrm{KN}$ and Hirano H, eds. Vocal Fold Physiology. Tokyo: Tokyo University Press, 1981:209-27.

18 Neilson PD, Andrews G, Guitar BE, Quinn PT. Tonic stretch reflexes in lip, tongue, and jaw muscles. Brain Res 1979; 178:311-27.

19 McRuer DT, Krendel ES. Human operator as a servo element pt. I, II. J Franklin Institute 1959;267:381-403, 511-536.

20 Poulton EC. Tracking Skill and Manual Control. New York: Academic Press, 1974.

21 Grahame D, McRuer DT. Analysis of Non-Linear Control Systems. New York: Dover Publ., 1971.

22 Bendat JS, Piersol AG. Measurement and Analysis of Random Data. New York: J Wiley and Sons, 1966.

23 Marmarelis PZ, Marmarelis VZ. Analysis of Physiological Systems. New York: Plenum Press, 1978.

24 Neilson PD, Neilson MD. Influence of control-display compatibility on tracking behaviour. $Q J$ of Exp Psychol 1980;132:125-35.

25 Winer BJ. Statistical Principles in Experimental Design. 2nd ed. New York: McGraw-Hill, 1971.

26 Barlow SM, Abbs JH. Orofacial fine motor control impairments in congenital spasticity: evidence against hypertonus-related performance deficits. Neurology 1984;34:145-50.

27 Neilson PD, Neilson MD, O'Dwyer NJ. Acquisition of motor skill in tracking tasks: learning internal models. In: Russell DG, Abernathy B, eds. Motor Memory and Control. Dunedin: Human Performance Associates, 1985:25-36. 